\title{
ENSINO E APRENDIZAGEM DE CÁLCULO I EM CURSOS DE LICENCIATURA: LIMITES E POSSIBILIDADES
}

\section{TEACHING AND LEARNING OF CALCULUS I IN UNDERGRADUATE DEGREE: LIMITS AND POSSIBILITIES}

\author{
Elcimar Simão Martins ${ }^{1}$ \\ Universidade da Integração Internacional da Lusofonia Afro-Brasileira \\ Damião Júnio Gonçalves Araújo ${ }^{2}$ \\ Universidade da Integração Internacional da Lusofonia Afro-Brasileira \\ Rodolfo Ferreira de Oliveira ${ }^{3}$ \\ Universidade da Integração Internacional da Lusofonia Afro-Brasileira
}

\begin{abstract}
Resumo
O Cálculo Diferencial e Integral é um ramo da Análise Matemática, essencial para o estudo da Matemática Pura, dos fenômenos da Natureza e também para a inovação tecnológica. É componente curricular obrigatório nos cursos de formação de professores na área das Ciências Exatas e da Natureza, tais como Matemática, Química, Física. É de extrema importância que estudantes desses cursos compreendam a essência do Cálculo para que logrem êxito nas disciplinas mais específicas no decorrer de seu curso. Entretanto, as disciplinas de Cálculo estão entre aquelas com maiores índices de reprovação nos cursos da área de exatas. Partindo dessa premissa, o presente artigo buscou investigar os limites e as possibilidades para o ensino e a aprendizagem de Cálculo I em cursos de licenciatura. A pesquisa se pautou na abordagem qualitativa, utilizando como instrumentos de coleta de dados a análise documental, entrevistas a docentes e aplicação de questionários a discentes do curso de Licenciatura em Ciências da Natureza e Matemática $(\mathrm{CNeM})$, no âmbito do Instituto de Ciências Exatas e da Natureza (ICEN), da Universidade da Integração Internacional da Lusofonia AfroBrasileira (UNILAB). Os resultados revelam que o maior responsável pelo mau desempenho da maioria dos estudantes nas disciplinas de Cálculo é devido a uma fraca base em Matemática desde a educação básica e que para compreender as ideias do Cálculo é necessário ter certa maturidade em teoria dos conjuntos, funções em geral e, principalmente, o conceito de função. $\mathrm{O}$ estudo revela ainda que para um ensino e aprendizagem de Cálculo I ser produtivo são necessários "n-fatores": que o professor objetive mais do que simplesmente cumprir a ementa, desenvolvendo as ideias básicas, de forma interativa, dinâmica e correlacionada com suas aplicações; que os estudantes se esforcem na disciplina e que haja a complementação de conceitos tidos como prérequisitos ao estudo do Cálculo.
\end{abstract}

Palavras-chave: Cálculo I; Cursos de Licenciatura; Ensino e Aprendizagem.

\footnotetext{
${ }^{1}$ Endereço eletrônico: elcimar@unilab.edu.br

${ }^{2}$ Endereço eletrônico: araujo@unilab.edu.br

${ }^{3}$ Endereço eletrônico: rodolfoyondaime26@gmail.com
} 


\begin{abstract}
Differential and Integral Calculus is a branch of Mathematical Analysis, essential for the study of models related to natural phenomena and technological innovation. It is a required curricular component for major undergraduate courses in natural sciences, such as Mathematics, Economics, Chemistry and Physics. It is of crucial importance that such students understand the essence of Calculus to succeed in the more specific topics to be approached in its courses. However, the Calculus subjects has one of the lowest academic results compared with undergraduate subjects. Based on this, the present article concerns to investigate the limits and possibilities for the teaching/learning of the Calculus I course. This study was based on the qualitative approach, using as data collection instruments documentary analysis, interviews with teachers and submitting questionnaires to students of the Undergraduate Degree in Ciências da Natureza e Matemática $(\mathrm{CNeM})$, under the supervision of the Instituto de Ciências Exatas e da Natureza (ICEN), from the Universidade da Integração Internacional da Lusofonia Afro-Brasileira (UNILAB). The results reveals us the main responsible for the general poor performance of such students: the weak knowledge base in Mathematics, since the basic education to a understanding necessary to the minimal requirements, as in particular the concept of a real function. Moreover, the study shows that for the teaching/learning to be productive, "n-factors" are necessary: more than simply to meet the related program schedule, is essential to develop the basic ideas, interactively, dynamically according to their applications; also, in font of the heavy mathematical language contained on Calculus study, students must to strive even more to obtain the needed complementation of concepts considered as pre requirements for this subject.
\end{abstract}

Keywords: Calculus I; Undergraduate Degree; Teaching and Learning.

\title{
Introdução
}

Concebido por Isaac Newton e Leibniz (de forma isolada), em meados do século XVII, o Cálculo Diferencial e Integral é um ramo da Análise Matemática, essencial para o estudo da Matemática Pura, dos fenômenos da Natureza e também para a inovação tecnológica. É componente curricular obrigatório nos cursos de graduação das ditas Ciências Exatas, tais como matemática, química, física e das diversas engenharias. Sendo assim, é de extrema importância que um estudante de um desses cursos consiga compreender a essência do Cálculo para ter sucesso no decorrer de seu curso nas disciplinas mais específicas.

Historicamente, as disciplinas de Cálculo estão entre aquelas com maiores índices de reprovação nos cursos de graduação, conforme estudos de Baroni (2015), Lima; Silva (2012), Godoy; Faria (2012), Santos; Matos (2012), dentre outros.

Para compreender as ideias do Cálculo é necessário ter certa maturidade em teoria dos conjuntos, funções em geral e, principalmente, no conceito de função. Apesar 
do conceito de função ter surgido após a elaboração do Cálculo, é essencial que, inicialmente, os alunos possuam um conceito formado de função como pré-requisito. Poderíamos pensar: a solução seria antes da disciplina de Cálculo existir uma disciplina que fizesse uma revisão e aprofundamento de Conjuntos e Funções, tal como um "PréCálculo". Somente isso não resolveria o problema, visto que na grade curricular dos cursos de: Física, Matemática, Engenharia de Energias, Química, existe uma ou mais disciplina(s) de Matemática precedente(s) ao Cálculo e que servem para revisar os conceitos de funções e conjuntos e mesmo assim há um grande índice de reprovação.

Poderíamos então pensar que o problema está no ensino de Cálculo. Embora saibamos que o docente possui uma parcela de responsabilidade não podemos atirar-lhe toda a culpa haja vista que há inúmeros fatores internos e externos à disciplina, ao curso e à Universidade que influenciam no ensino e na aprendizagem. Dependendo do curso em que a disciplina está sendo ministrada é necessário que a abordagem seja diferenciada, preparando o indivíduo para as suas disciplinas específicas, para que saibam como e onde aplicar a teoria do cálculo. Por exemplo, um estudante de Física, deve ver o Cálculo a partir de suas aplicações na Mecânica, no Eletromagnetismo, na Termodinâmica, precisando fixar, por exemplo, o conceito de taxa variação, algo essencial ao estudo dos fenômenos naturais. Já um estudante de Matemática necessita ver o Cálculo mais aprofundado que os demais, dialogando sempre com a Topologia e a Análise Real para que seja preparado para as cadeiras seletas do seu curso.

O professor de Cálculo precisa lecionar de acordo com os objetivos do seu público-alvo, adequando suas metodologias para abranger as necessidades de cada curso em relação à disciplina. Surge outro questionamento: é possível a aula de Cálculo ser dinâmica, interativa? Claramente que sim. Um recurso é o uso das chamadas Tecnologias da Informação e Comunicação (TICs), como o software GeoGebra, o qual é utilizado por alguns docentes em cursos de licenciatura, principalmente na resolução dos exercícios, em que o software permite visualizar, por exemplo, a geometria por trás do Cálculo.

A pesquisa se pautou na abordagem qualitativa, utilizando como instrumentos de coleta de dados a análise documental, entrevistas a docentes e aplicação de questionários a discentes do curso de Licenciatura em Ciências da Natureza e Matemática (CNeM), no âmbito do Instituto de Ciências Exatas e da Natureza (ICEN), da Universidade da Integração Internacional da Lusofonia Afro-Brasileira (UNILAB). 
Foram analisados o Projeto Pedagógico do Curso CNeM, assim como cinco planilhas de notas da disciplina Cálculo I. Foram entrevistados dois docentes que ministram/ministraram a disciplina e dezoito estudantes responderam a um questionário a partir de um formulário eletrônico. Os dados coletados foram analisados à luz de vários teóricos e compõem os próximos tópicos.

\section{O ensino e a aprendizagem de Cálculo I em um Curso de Licenciatura}

Em geral, na maioria dos cursos, na disciplina de Cálculo 1, a primeira aula começa da seguinte maneira. Seja $f: A \subseteq \mathbb{R} \rightarrow \mathbb{R}$. Dizemos que $f$ é contínua em $x_{0} \in A$, se $\forall \epsilon>0, \exists \delta>0$, tal que, se:

$$
0<\left|x-x_{0}\right|<\delta \rightarrow\left|f(x)-f\left(x_{0}\right)\right|<\epsilon
$$

E, portanto:

$$
\lim _{x \rightarrow x_{0}} f(x)=f\left(x_{0}\right)
$$

Acreditamos que um leitor leigo no assunto provavelmente não compreenda o que está expresso acima, mas o que queremos mostrar é que se um indivíduo não possuir uma determinada maturidade nos operadores lógicos e símbolos matemáticos como poderá ele compreender e atribuir sentido ao que está sendo dito nessa sentença?

De acordo com Vygotski (1991), o indivíduo constrói e atribui significados a um signo a partir da realidade e contexto social em que se encontra inserido, sendo fortemente influenciado pela cultura. A partir desse ponto de vista podemos ver que um estudante que nunca foi habituado com tais notações, conceitos, simbologias e operadores não poderá atribuir significados para tal definição e não será capaz de compreender essa sentença, nem tudo o que está por trás dela.

Como podemos solucionar esse problema? Ora, via de regra, ao estudante não é apresentada uma matemática que explore tais funções cognitivas. Pelo contrário, a ele geralmente é apresentada uma matemática de regras de "decoreba", de aplicações de fórmula, sem muitas vezes não se ter ao menos noção do que tal equação representa. Voltamos à pergunta: Como solucionar esse problema? Acreditamos que para solucionar tal problema, altamente recorrente em toda a sociedade brasileira, o correto 
seria abordar a matemática e sua linguagem desde os primeiros anos do ensino fundamental.

De acordo Nascimento e Santo (2013) em um estudo revelando as potencialidades cognitivas das crianças, em especial sua facilidade em aprender uma segunda língua:

a criança pode ser exposta a uma gama variável de novos conhecimentos, visto que seu desenvolvimento cerebral, surpreendentemente acelerado nos primeiros cinco anos de vida, impõe uma rede sináptica bastante ramificada e extensa, que vai sendo reduzida com o passar do tempo, em virtude da seleção natural de conexões neuronais relacionada com a necessidade básica de utilização das conexões mais imediatas e apropriadas ao que o ambiente requer (p. 19).

A capacidade humana de aprender uma segunda língua, no nosso caso, a linguagem matemática possui um pico durante a primeira infância e ao passar do tempo, vai caindo gradativamente, por isso, um estudante ao qual nunca lhe fora apresentado a linguagem matemática, provavelmente não conseguirá compreendê-la ou terá mais dificuldades para tal quando estiver cursando Cálculo, mesmo que haja disciplinas preparatórias que venham a trabalhar os conteúdos tidos como pré-requisitos.

De acordo com Piaget (1970), a aprendizagem se dá a partir da construção de esquemas e à medida que a criança vai envelhecendo, suas possibilidades cognitivas se alteram, ou seja, o desenvolvimento da criança, a partir dos quatro estágios - sensóriomotor, pré-operatório, operatório-concreto e operatório-formal - é o que molda a sua aprendizagem. Tais estágios são preenchidos de acordo com o contexto em que a criança está inserida.

Ainda segundo Piaget (1971), a partir do estágio operatório concreto (7 aos 11 anos) surge na criança a capacidade de fazer análises lógicas, como por exemplo: se A é maior que B, e B é maior que C, então A é maior que C. Ou seja, essa fase seria a ideal para introduzir no estudante a linguagem matemática, através dos operadores e sentenças lógicas, tais como "se então", "se e somente se", entre outros.

Partindo dessa perspectiva, buscamos compreender os limites e as possibilidades de ensino e aprendizagem de Cálculo I, no âmbito do curso de Licenciatura em Ciências da Natureza e Matemática ( $\mathrm{CNeM}$ ), vinculado ao Instituto de Ciências Exatas e da Natureza (ICEN), da Universidade da Integração Internacional da Lusofonia AfroBrasileira (UNILAB), que foi criada pela Lei $\mathrm{N}^{\circ} 12.289$, de 20 de julho de 2010, com o 
objetivo de formar recursos humanos visando à integração entre o Brasil e os demais países membros da Comunidade dos Países de Língua Portuguesa (CPLP), assim como favorecer o desenvolvimento regional e o intercâmbio cultural, científico e educacional.

A Unilab conta com três campi entre os municípios de Redenção e Acarape (Ceará/Brasil) e um campus em São Francisco do Conde (Bahia/Brasil). A instituição oferta cursos voltados para áreas de interesse do Brasil e dos países parceiros, reunindo estudantes e docentes brasileiros e internacionais com o objetivo de contribuir para a superação das desigualdades, representando um avanço na cooperação e internacionalização do Ensino Superior (BRASIL, 2010). Atualmente, são ofertados cursos de Licenciatura em Ciências da Natureza e Matemática, Física, Química, Matemática, Ciências Biológicas, História, Letras, Pedagogia, Sociologia, além dos Bacharelados em Humanidades, Antropologia, Administração Pública, Agronomia, Enfermagem e Engenharia de Energia.

A instituição ${ }^{4}$ conta com um total de 4.726 estudantes, distribuídos em cursos de graduação e pós-graduação, presencial e a distância. Nos cursos de graduação presencial são 3.398 estudantes, sendo 2.510 brasileiros e 888 internacionais. Ainda há 481 estudantes em cursos de Graduação a distância. Na pós-graduação há 847 estudantes, sendo 686 matriculados em cursos de especialização a distância e 71 em cursos de mestrado.

O Curso de Licenciatura em Ciências da Natureza e Matemática tem o objetivo de formar docentes para "atuar no Ensino Fundamental, além de possuir habilitação específica para atuar no Ensino Médio, em uma das seguintes disciplinas: Química, Física, Biologia ou Matemática, em instituições públicas e privadas” (UNILAB, 2010, p. 1). O CNeM é divido em duas partes: formação comum (com 2.250 horas) e formação específica nas habilitações (com 810 horas) de Biologia, Física, Matemática ou Química, de acordo com a escolha do estudante.

Analisamos cinco planilhas de notas da disciplina Cálculo I referente aos trimestres: 2012.2, 2012.3, 2013.2, 2014.1 e 2015.1 do CNeM. Nos parágrafos seguintes detalhamos os resultados obtidos.

No trimestre de 2012.2 foram matriculados vinte e seis estudantes na referida disciplina. Desse total, treze foram aprovados e treze foram reprovados. Três estudantes

\footnotetext{
${ }^{4}$ Conforme dados de novembro de 2016 da Diretoria de Registro e Controle Acadêmico - DRCA, disponíveis no sítio oficial da Unilab $<$ http://www.unilab.edu.br $>$.
} 
se submeteram à avaliação final, mas apenas um logrou êxito. O índice de aprovação foi de $50 \%$, sendo que $46,15 \%$ tiveram aprovação direta.

Em 2012.3 trinta e nove estudantes matricularam-se na disciplina. Trinta e sete foram aprovados e dois reprovados. Do total de aprovados, vinte e um conseguiram êxito após terem feito a avaliação final. O índice de aprovação geral foi de 94,87\%, sendo que apenas $43,25 \%$ tiveram aprovação direta.

No trimestre de 2013.2 foram matriculados vinte e cinco estudantes na disciplina. Dezessete foram aprovados e oito reprovados. Do total, seis se submeteram à avaliação final, mas somente quatro conseguiram a aprovação. O índice de aprovação geral foi de $68 \%$, sendo que apenas $52 \%$ tiveram aprovação direta.

Em 2014.1 foram matriculados trinta e seis estudantes na disciplina. Vinte e quatro foram aprovados e doze reprovados. Do total, dezenove se submeteram à avaliação final, onze conseguiram a aprovação e oito ficaram reprovados. O índice de aprovação geral foi de $66,6 \%$, sendo que apenas 36,1\% tiveram aprovação direta.

No trimestre de 2015.1 trinta e quatro estudantes se matricularam na referida disciplina. Doze estudantes foram aprovados e vinte e dois reprovados. Quatro estudantes se submeteram à avaliação final e três conseguiram êxito. $O$ índice de aprovação geral foi de 35,29\%, sendo que apenas 26,47\% tiveram aprovação direta.

Analisando as cinco ofertas, verificamos que somente em um trimestre mais da metade da turma passou por média sem necessidade de avaliação final. $\mathrm{O}$ trimestre 2012.3 foi o que teve o maior percentual de estudantes aprovados e o de 2015.1 foi o que teve o menor percentual de aprovados.

Uma das causas que avaliamos para o grande índice de reprovação em Cálculo é a maioria dos alunos chegar do ensino médio com uma fraca base em matemática, em conteúdos tidos como pré-requisitos, como funções e teoria dos conjuntos. Porém, a maior deficiência apresentada pelos estudantes é a falta de capacidade abstrativa, em especial uma dificuldade em entender as simbologias matemáticas e os operadores lógicos essenciais para a construção das sentenças, tais como: teoremas, proposições, definições, axiomas, entre outros, o que é fundamental para a compreensão do Cálculo. Os estudos de Santos e Matos (2012) revelam que tais fatores têm sua responsabilidade nos resultados da disciplina, haja vista que todos eles fazem parte do processo de ensino e aprendizagem de Cálculo. 


\section{Cálculo I em cursos Licenciatura: o que dizem os professores}

Buscando compreender os limites e as possibilidades do Cálculo I no Curso de Licenciatura em Ciências da Natureza e Matemática da Unilab, entrevistamos um professor e uma professora que ministram/ministraram a referida disciplina e para fins de análise nesse texto serão chamados de docente A e de docente B. Os sujeitos têm doutorado e mestrado em Física. Um deles tem licenciatura em matemática e outro é bacharel em Física e estão na faixa etária entre trinta e quarenta anos.

Como já exposto, o Cálculo fundamenta sua importância nas suas mais variadas aplicações, tanto em Matemática, como em Física, Química e até mesmo Biologia, por isso é necessário que o docente "diga qual o objetivo da disciplina e quais são suas aplicações. É extremamente importante que no primeiro dia o aluno saia de sala ciente de que o Cálculo I é a base para a construção de todas as ciências exatas e das engenharias, por exemplo", como disse o docente A em sua fala.

$\mathrm{Na}$ linha de raciocínio de mostrar as aplicações do que se está ensinando, Machado (2009) nos leva a refletir sobre a consciência do professor de matemática em sala de aula, enfatizando na célebre pergunta que todo professor que já ministrou aulas dessa área certamente já ouviu: "para que serve isso?".

Saber a funcionalidade daquilo que está sendo estudado contribui para a chamada aprendizagem significativa, discutida por Ausubel $(1963,1968)$, ao propor que o conteúdo lecionado seja de fato revelador para quem está sendo ensinado, para que ele sinta-se mobilizado a relacionar o que é dito e o que é visto na prática.

O referido pesquisador enfatiza a importância de ensinar sobre o que estudante já tem como conhecimento prévio, para que o novo conhecimento possa usar os anteriores como alicerce. O problema é quando o estudante não possui uma boa base, que é o acontece com a maioria dos estudantes quando se deparam com o Cálculo I, como ressalta o Professor A: "se queremos realizar um estudo mais aprofundado das funções, devemos já ter algum conhecimento prévio sobre elas”. Ainda nessa pauta dos desafios e dificuldades, que foi o tema mais enfatizado pelo docente $\mathrm{B}$, que ressalta várias questões aqui já discutidas, como a falta de base; turma composta por poucos alunos com bons conhecimentos prévios e uma boa quantidade sem conhecimento prévio; alguns alunos dedicados e mobilizados e alunos que apenas "fazem a disciplina 
por fazer", justamente por não verem sentido em estudar algo que a priori não terá influências em sua profissão, tampouco em sua vida.

Outra dificuldade, que foi também relatada pelos dois professores é a ocorrência da disciplina no Regime Trimestral, aliada ao fato de que no período que ocorre a disciplina de Cálculo I no Curso de Ciências da Natureza e Matemática, ocorrem simultaneamente outras sete disciplinas, deixando o tempo para estudo muito reduzido, o que traz como resultado "atraso no andamento da disciplina e alto índice de reprovação”, como diz o docente $\mathrm{B}$.

Para solucionar o problema de falta de base, o docente A propôs a "realização de um curso de pré-cálculo para sanar as deficiências do ensino médio em funções”, mas isso já acontece, pois nos três primeiros períodos do curso de $\mathrm{CNeM}$ existe um ramo de disciplinas configuradas como Fundamentos de Matemática, que servem justamente para preparar os estudantes para o curso de Cálculo e inclusive o docente B ressalta que sempre busca "iniciar a disciplina devagar, tentando preencher, na medida do possível, as lacunas advindas do ensino médio”. O que há de errado então?

Segundo o docente B, o maior problema é que, geralmente, os estudantes não compreendem que o sistema na Universidade é completamente diferente do sistema no Ensino Médio, "no sentido de que deveria haver um amadurecimento em relação às suas atitudes e posturas como estudantes universitários”, pois os estudantes ainda estavam muito dependentes daquilo que o professor passava na sala de aula e não procuravam o conhecimento por si próprio através de exercícios do livro, por exemplo. Ainda de acordo com o docente B, "matemática, além de tudo, é também, praticar".

Claro que o professor tem sim grande responsabilidade no desempenho de seus estudantes no decorrer da disciplina, pois muitas vezes o ensino não é aliado à prática, ao real e também poucos professores tentam utilizar as TICs em suas aulas (CANTINI, 2006).

\section{Cálculo I em cursos Licenciatura: o que dizem os estudantes}

A partir das planilhas de notas de Cálculo I disponibilizadas pela coordenação do $\mathrm{CNeM}$, entramos em contato com estudantes que já cursaram a referida disciplina, buscando compreender os limites e as possibilidades para o ensino e a aprendizagem de Cálculo I no citado curso. 
Dezoito estudantes responderam ao formulário eletrônico. Desse total, onze são brasileiros e sete são estudantes internacionais. Catorze se identificaram como do sexo masculino e quatro do feminino. Cinco estão na faixa etária entre 18 e 20 anos. Doze estão entre 21 e 25 anos. Apenas um dos sujeitos tem mais de trinta anos. Todos os brasileiros cursaram o ensino médio na rede pública de ensino e três internacionais cursaram na rede privada, revelando a importância da Universidade como veículo de inclusão social.

Em um curso de graduação o estudante se depara com diversas disciplinas e, como é o caso do Curso de Ciências da Natureza e Matemática, das mais variadas áreas do conhecimento. É evidente que todas as disciplinas possuem sua importância tanto na qualificação do indivíduo quanto em sua formação pessoal, mas também é claro que algumas destas refletem mais nos objetivos do aluno e em suas perspectivas, fazendo com que ele tenha um maior apreço por elas.

Os estudantes foram indagados em relação à aprovação na disciplina. O gráfico presente na Figura 1 esboça esse resultado:

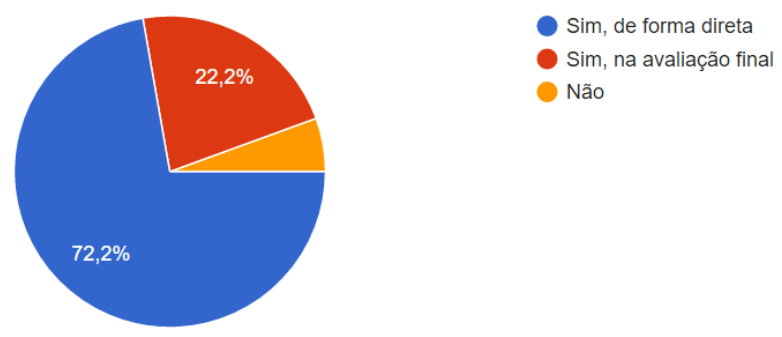

Figura 1 - Gráfico de aprovação em Cálculo I (sujeitos da pesquisa)

O gráfico revela que mais de $70 \%$ dos sujeitos que participaram da pesquisa foram aprovados na disciplina de Cálculo I sem necessidade de avaliação final, dado bem distinto do encontrado na análise das planilhas de nota, que revelaram uma média de $40 \%$ de aprovação.

Nessa pauta, questionamos aos estudantes qual o valor da disciplina de Cálculo I para a sua formação (pessoal e profissional) e tivemos um bom contraste de respostas: a grande maioria citou como a principal importância dessa disciplina o preparo para os demais cálculos e também as disciplinas restantes do seu curso, em especial nas habilitações de Matemática e Física, como é o caso do Estudante 3: "Essa disciplina 
teve valor fundamental para minha formação, pois me serviu de alicerce para as demais".

Houve também alguns alunos que enfatizaram a importância da disciplina em suas aplicações e nas possibilidades que tal curso permite, como relata o Estudante 5:

É uma experiência única e significativa, indispensável para a minha formação. É nessa disciplina que encontramos os conceitos fundamentais que permanecerão conosco durante todo o curso $e$, quem sabe, durante a vida. O conceito de derivada, integral, o teorema fundamental do Cálculo, dentre outros. Possivelmente o mais importante não tenha sido aprender as técnicas de derivar e integrar, mas acima de tudo o conceito e a ampliação do poder de abstração.

Uma fala em especial nesse comentário nos chamou a atenção em particular, quando vemos o real objetivo das disciplinas de Cálculo, que não é aprender técnicas de derivação ou integração e sim ampliar o poder da abstração, bem como direcionar os estudantes para o que os matemáticos chamam de "Matemática de verdade".

Não obstante, houve sim relatos de estudantes que não viram e que ainda não veem importância no estudo dessa disciplina para a sua formação, como é o caso do Estudante 11: "Para mim nem influiu e nem contribuiu. Ainda quero saber onde vou usar limites e derivadas na minha profissão". Essa realidade se deve porque provavelmente não foram apresentadas a este aluno as aplicações que o Cálculo possui em sua área de formação, que mais adiante ele diz que é Biologia, e se isso não acontece como pode o estudante criar algum valor para essa disciplina? Como a aprendizagem do indivíduo poderá ser significativa se o mesmo não vê razão para realizar tal curso? (AUSUBEL, 1963; 1968).

Mesmo vários desses estudantes tendo feito a disciplina juntos, na mesma turma, as experiências e aprendizagem de todos são bem particulares e isso vale para qualquer grupo social. Nessa perspectiva, buscamos questionar como foi a experiência de cada aluno na disciplina e, no geral, avaliamos que foi boa, salvo alguns casos particulares, como é o caso do Estudante 8, ao relatar que a "experiência no Cálculo 1 não foi tão boa", devido uma troca de professor no decorrer na disciplina e com a metodologia do novo professor, tal aluno não conseguia aprender como com o anterior e então acabou indo para a Avaliação Final, mas lá conseguiu aprovação na disciplina.

Dois estudantes relataram que a experiência não foi tão boa quanto o esperado, pois a abordagem foi bastante superficial e rasa em conteúdos, causa que supomos ser a 
ocorrência da disciplina, a qual possui uma vasta ementa, em regime trimestral, o que impede uma abordagem mais focalizada e força muitas vezes o professor a dar mais atenção a alguns conteúdos por ele classificados como mais importantes e essenciais.

Já doze estudantes apontaram a disciplina como "boa", "muito boa" ou "excelente" e um relato do Estudante 1 reflete a importância do Cálculo para o estudo da Natureza e principalmente para a sua formação, pois "mudou minhas percepções quanto à natureza abstrata da matemática, bem como minhas percepções sobre os fenômenos naturais e mostrou como o Cálculo é uma essência da Natureza"

A vida, como sabemos, é feita de desafios e isso não é diferente em um curso de graduação, tampouco uma disciplina de Cálculo I e tendo isso em mente questionamos os estudantes quanto aos maiores desafios e obstáculos encontrados no decorrer da disciplina, os quais são naturais e recorrentes nos processos de ensino e aprendizagem. Dois dos respondentes afirmaram que não tiveram desafios e dificuldades durante a disciplina, mas os outros dezesseis relataram diversas adversidades, as quais em sua maioria se revelam na ocorrência da disciplina em regime trimestral, aliada ao fato de serem executadas várias disciplinas no mesmo período, o que reduz drasticamente tanto o tempo para o ensino, quanto o tempo para a aprendizagem, deixando assim um curso debilitado e provavelmente que não se faz suficiente para as necessidades do estudante.

Apesar de tudo isso, o Estudante 5 nos fez um relato totalmente diferente dos demais, elencando que o principal problema não era nem a complexidade dos conteúdos, nem a rapidez com que a disciplina ia se consolidando e sim os estudantes que cursaram a disciplina anteriormente que ficavam utilizando discursos negativos quanto ao curso e que poderiam criar uma barreira no estudante para cursar a disciplina, vendo-a como um obstáculo antes mesmo de iniciá-la:

O maior desafio foi escutar o discurso dos alunos que já fizeram essa disciplina anteriormente, uma vez que o mesmo era recheado de terror e pessimismo. Eles diziam que a disciplina só servia para reprovar e que eu devia estar preparado que também reprovaria. E além disso denegriam a imagem dos professores dessa disciplina. Ouvir essas coisas foi o mais difícil. Mas quando fui participando das aulas acabei vendo que não era bem assim e que bastava tentar suprir minhas deficiências de formação do ensino médio para poder ter um rendimento satisfatório na Universidade. Aprendi a nunca falar mal de disciplinas ou de professores para os alunos que não tiveram ainda aulas destas disciplinas ou desses professores. Meu posicionamento quando sou indagado é 'vá lá e faça seu melhor, tire suas próprias conclusões' (Estudante 5). 
Desafios são inerentes ao processo de ensino e aprendizagem, mas possibilidades também, refletindo a essência do conhecimento e a importância da educação na sociedade. Não obstante, o Cálculo abre um leque de possibilidades para o indivíduo de um curso de Exatas, pois permite estudar detalhadamente as funções através de conceitos como limite, derivada e integral e se fazem bastantes úteis no decorrer de toda a graduação do discente, seja ele da Matemática, Física, Química, Engenharia ou da Biologia, como por exemplo, no estudo do crescimento e variação populacional de determinada espécie. Quando o estudante 11 afirma que é da habilitação em Biologia e que até agora não viu contribuições da disciplina em sua formação significa que o professor não se preocupou em mostrar as aplicações que tal setor de estudo possui em todas essas áreas, limitando as possibilidades do estudante, que de acordo com o Estudante 9 permitiram "compreender e adquirir ferramentas matemáticas essenciais para minha formação".

Tendo essa discussão em vista, nos questionamos: como poderia então ser ministrada essa disciplina, em especial em um curso multidisciplinar e com estudantes de vários países, várias culturas e diferenciadas visões de mundo? Uma alternativa é o uso das chamadas Tecnologias da Informação e Comunicação (TICs) nas aulas, em especial do GeoGebra, como foi sugerido pelos estudantes 1, 2, 5 e 9, pois tais recursos "deixa[m] a aprendizagem do estudante mais significativa" (Estudante 1) e assim voltamos ao discutido no início. O referido software permite representar gráficos de funções de uma e duas variáveis, figuras e sólidos geométricos, curvas parametrizadas, entre outras coisas, o que facilita a visualização dos conceitos vistos em sala, favorecendo assim a aprendizagem do estudado.

Ainda há muitas outras possibilidades para o desenvolvimento dessa disciplina e que se aplica a muitas outras, como vemos na fala do Estudante 9, quando cita a importância da "contextualização histórica do desenvolvimento do Cálculo, para que os estudantes percebam que a Ciência não surge do nada, de uma 'tábula' rasa" e do uso de "avaliações contínuas e não pontuais, e em um formato para além das provas comuns".

Mesmo com todas essas possibilidades elencadas ainda assim vemos que há muito a ser feito para melhorar a realidade das disciplinas de Cálculo nos cursos de licenciatura, pois cada um dos "n-problemas" verificados precisa de mecanismos 
eficazes para serem resolvidos e tais soluções dependem de uma colaboração tanto dos alunos, quanto dos professores e até mesmo de faores externos.

\section{Considerações}

O Cálculo I é componente curricular obrigatório nos cursos de formação de professores na área das Ciências Exatas e da Natureza, tais como Matemática, Química, Física, sendo fundamental que estudantes desses cursos compreendam a sua essência para que logrem êxito nas disciplinas mais específicas no decorrer de seu curso.

A análise das planilhas de notas das disciplinas de Cálculo I no âmbito do CNeM revela a dificuldade que muitos estudantes enfrentam para aprovação direta na referida disciplina.

Os professores entrevistados ressaltam a importância de o docente deixar claro para a turma os objetivos e as aplicações da disciplina. Um limite apresentado é o fato de boa parte dos estudantes não apresentarem boa base em relação à matemática. Outra dificuldade é trabalhar toda a disciplina de maneira condensada em um trimestre.

Os estudantes compreendem que o Cálculo é importante como base para os demais cálculos e também para as demais disciplinas do curso e mais de $70 \%$ dos sujeitos da pesquisa consideraram positiva a experiência com a disciplina. Poucos estudantes não conseguiram encontrar importância na disciplina. Quase a totalidade dos respondentes revelou, assim como os professores, a dificuldade de cursar a disciplina no regime trimestral, o que reduz o tempo para o ensino e a aprendizagem, haja vista que ainda cursam em média mais sete disciplinas. As TICs, e em especial o software GeoGebra, foi apresentado como uma possibilidade para o desenvolvimento da disciplina.

Para o Cálculo I poder ser produtivo para os estudantes são necessários "nfatores", tais como: que o professor objetive mais do que simplesmente cumprir a ementa da disciplina, desenvolvendo as ideias básicas, de forma interativa, dinâmica e correlacionada com suas aplicações; que os estudantes se esforcem na disciplina e que haja a complementação de conceitos tidos como pré-requisitos ao estudo do Cálculo. É fundamental que os docentes trabalhem com o Cálculo I de modo que os estudantes operem logicamente com conteúdos básicos à sua formação em um curso da área das exatas, sobretudo os que estão na licenciatura. 


\section{Referências}

AUSUBEL, D. P. The psychology of meaningful verbal learning. New York, Grune and Stratton, 1963

Educational psychology: a cognitive view. New York, Holt, Rinehart and

Winston, 1968

BARONI, R. L. S. Algumas questões sobre o ensino de análise em cursos de formação de professores de matemática. III Fórum de Discussão Parâmetros Balizadores da Pesquisa em Educação Matemática no Brasil. São Paulo: PUC/SP, 2015.

BRASIL. Lei $\mathbf{N}^{\mathbf{0}} \mathbf{1 2 . 2 8 9}$, de 20 de julho de 2010. Dispõe sobre a criação da Universidade da Integração Internacional da Lusofonia Afro-Brasileira - UNILAB e dá outras providências. Brasília: Casa Civil, 2010.

CANTINI, M. C., et al. O desafio do professor frente as novas tecnologias. VI EDUCERE - Congresso Nacional de Educação. PUC-PR. Curitiba, 2006. pp. 875-883

LIMA, G. L.; SILVA, B. A. O ensino do cálculo na graduação em matemática: considerações baseadas no caso da USP. V Seminário Internacional de Pesquisa em Educação Matemática, 2012, Petrópolis, Rio de janeiro. Anais do $5^{\circ}$ Seminário Internacional de Pesquisa em Educação Matemática. Brasília: SBEM, 2012.

MACHADO, N. J. Matemática e Realidade. 7. ed. São Paulo: Cortez, 2009.

NASCIMENTO, D. C.; SANTO, E. E. O despertar da segunda língua na primeira infância: uma análise sob a perspectiva neuropsicológica. Cadernos Intersaberes, vol. 1, n 2, Curitiba: UNINTER, 2013. pp. 18-37.

PIAGET, J. Epistemologia genética. São Paulo: Martins Fontes, 1970.

Seis estudos de psicologia. Rio de Janeiro: Forense Universitária, 1971.

SANTOS, S. P.; MATOS, M. G. O. O ensino de cálculo i no curso de licenciatura em matemática: obstáculos na aprendizagem. In: Revista Eventos Pedagógicos. v. 3, n. 3, p. 458 - 473, Ago. - Dez. 2012.

UNILAB. Projeto Político Pedagógico do Curso de Ciências da Natureza e Matemática - licenciatura. Redenção/CE, 2014.

Resolução $\mathbf{N}^{\circ}$ 02/Conselho Superior Pró Tempore da Unilab, em 18 de Novembro de 2010. Redenção/CE, 2010.

VYGOTSKI, L. S. A formação social da mente. 4. ed. São Paulo: Livraria Martins Fonseca, 1991. 